\title{
Editorial: Nutritional Intervention for the Intestinal Health of Young Monogastric Animals
}

\author{
Rajesh Jha ${ }^{1 *}$ and Sung Woo Kim ${ }^{2 *}$ \\ ${ }^{1}$ Department of Human Nutrition, Food and Animal Sciences, University of Hawaii at Manoa, Honolulu, HI, United States, \\ ${ }^{2}$ Department of Animal Science, North Carolina State University, Raleigh, NC, United States
}

Keywords: antimicrobial resistance, gut health, prebiotics, probiotics, pig, poultry

Editorial on the Research Topic

Nutritional Intervention for the Intestinal Health of Young Monogastric Animals

\section{INTRODUCTION}

Poultry and pig production have increased at a faster rate than any other livestock production globally (1). Among others, nutritionally balanced-feeding programs, along with antibiotic growth promoters (AGP) in feeds, played a significant role in achieving this success $(2,3)$. The animal industry, however, aims to redefine its nutrition program to grow safe and quality meat in the

OPEN ACCESS

Edited by:

Domenico Bergero,

University of Turin, Italy

Reviewed by:

Claudio Forte

University of Turin, Italy

*Correspondence:

Rajesh Jha

riha@hawaii.edu

Sung Woo Kim

sungwoo_kim@ncsu.edu

Specialty section:

This article was submitted to Animal Nutrition and Metabolism,

a section of the journal

Frontiers in Veterinary Science

Received: 16 February 2021

Accepted: 24 February 2021

Published: 18 March 2021

Citation:

Jha R and Kim SW (2021) Editorial:

Nutritional Intervention for the

Intestinal Health of Young Monogastric

Animals. Front. Vet. Sci. 8:668563.

doi: 10.3389/fvets.2021.668563 light of public health concerns due to the use of AGP in diets (1). Maintenance or improvement of intestinal health is essential for optimum growth, better feed efficiency, and the overall health of pigs and poultry (4-6). Keeping a healthy intestine is also critically important for nutrient digestion and utilization, thereby ensuring better growth performance of pigs and poultry (7-9).

Intestinal health covers efficient nutrient utilization, macro- and micro-structural integrity of the gut, the stability of the microbiota, and the status of the immune system $(4,10,11)$. Moreover, intestinal health is a complex field combining the nutrition, microbiology, immunology, and physiology of animals. Challenges in intestinal health directly influence nutrient digestion and absorption $(4,12,13)$, which in turn reduces feed efficiency and increases susceptibility to enteric diseases $(14,15)$.

Recent regulatory changes on the use of AGP and selected feedstuffs have challenged the optimal growth and health of modern pigs and poultry, that have been extensively selected for growth efficiency and lean gain. Highly lean and fast-growing pigs and poultry highlight the need for a better understanding of the gut function and overall gut health. Understanding and improving the intestinal health of animals is a key essential trend needed for the success of animal production in this era of AGP free production $(1,4,16)$. This Research Topic eBook covers nutritional aspects of improving the intestinal health of monogastric animals, including current challenges and potential solutions. The papers have been presented under two sections: (1) Importance and understanding intestinal health of monogastric animals and (2) Nutritional intervention for intestinal health.

\section{IMPORTANCE AND UNDERSTANDING THE INTESTINAL HEALTH OF YOUNG MONOGASTRIC ANIMALS}

It is well-established that effective modulation of the gut health parameters depends not only on feedstuffs but also on the methods and timing of the nutrients available to host animals. Furthermore, early growth and development of GIT are of critical importance in enhancing 
nutrient utilization and optimizing the growth of poultry. Early nutrition programming using both in ovo and post-hatch feeding has been used as a means to modulate the early growth and development of GIT and has been found to be an effective strategy [(17); (Jha, Singh et al.)]. Similarly, the weaning phase of pigs is an incredibly stressful period as it causes morphological and functional changes in the gut and induces post-weaning growth depression. Different nutritional strategies, including the addition of functional feed additives in the weaner pig's diet have been proposed to minimize these effects (Zheng et al.). Similarly, different nutrients and feed additives have been used to optimize the gastrointestinal integrity and immune system of young animals (Adedokun and Olojede). However, gut microbiota plays a significant role in managing the gut environment by producing fermentation metabolites and influencing nutrient utilization pathways (18). Thus, it is important to understand the gut microbial ecology in-depth, including their taxonomic composition and biochemical functions. However, the gut microbiota is primarily influenced by diet, age, species, and location in the digestive tract $(5,18,19)$. Different techniques have been used to characterize the gut microbiota, but those have different strengths and limitations. Modern techniques like $16 \mathrm{~S}$ rRNA based next-generation sequencing and others are powerful tools to investigate the biological and ecological roles of the gut microbiota [(19); (Shang et al.)]. In the commercial animal production system, different nutritional and environmental stresses and pathological factors create oxidative stress in animals, leading to imbalances in the intestinal homeostasis due to the generation of reactive oxygen species (ROS) and reactive nitrogen species. It can be mitigated by supplementing exogenous vitamins, antioxidants, and plant extracts that have antioxidant properties that scavenge ROS [(9); (Mishra and Jha)]. Thus, it is crucial to understand the involvement of oxidative stress in the gastrointestinal functionality of animals and the potential intervention strategies available to maintain redox balance in the GIT.

\section{NUTRITIONAL INTERVENTION FOR INTESTINAL HEALTH}

It is not only the type of feedstuffs, but also their forms that have been found to affect gut health and function. A finer feed particle size enables optimal nutrient utilization and enhances animal performance due to increased surface area, allowing for better contact with digestive enzymes. Moreover, adequate diminution of feedstuffs is beneficial to feed manufacturing processes such as mixing and hydrothermal treatments, including pelleting, extrusion, and expansion. Thus, feed processing techniques, along with the type of feedstuffs, need to be considered when formulating diets for animals considering their impact on intestinal health (Kiarie and Mills).

As various feedstuffs, their components, and feed additives behave and function differently in the GIT of animals, different feeding strategies have been tested, with some success, to improve intestinal health and functionality. Furthermore, there is also a need to evaluate potential alternatives to AGPs in animal diets in the post-antibiotic era [(3); (Yang et al.)]. As potential alternatives to AGPs, different dietary fibers (DF), prebiotics, probiotics, postbiotics, enzymes, and others have been evaluated and found to have promising outcomes (Zheng et al.). Although DF are not well-digested and are often considered as anti-nutritional factors in monogastric animals, as it reduces nutrient utilization (20), it has been widely used in recent years to modulate the intestinal environment (4). DFs are fermented in the intestine and become short-chain fatty acids, stimulating the growth of health-promoting gut bacteria, and boosting the immune system [(21); (Jha, Fouhse et al.)]. In addition, specific nutrients such as functional amino acids like arginine, cysteine, glutamine, or glutamate, may enhance intestinal mucosa immunity, reduce oxidative damage, stimulate proliferation of enterocytes, and enhance the gut barrier function of weaned pigs (Xiong et al.). Amino acids, which are major nutrients for monogastric animals, are not only obligatory for maintaining the intestinal mucosal mass and integrity, but also for supporting the growth of microorganisms in the gut. Dietary amino acids are the major fuel of the small intestinal mucosa. Particularly, glutamate, glutamine, and aspartate are the primary oxidative fuel of the intestine (Yang and Liao). Trace minerals like copper, zinc, iron, and manganese have also been found to influence gut health parameters (Shannon and Hill). For example, pharmacological concentrations of copper have been shown to enhance growth, while high concentrations of zinc fed to newly weaned nursery pigs reduced the incidence of diarrhea from the proliferation of enterotoxigenic Escherichia coli and Clostridium and improved gut morphology. As a potential alternative to AGPs, prebiotics including mannan oligosaccharides, b-glucans, and fructans, are gaining more attention to be used in monogastric feeding program as prebiotics have been found to modulate microbial communities and regulate the production of cytokines and antibodies, improving gut development and the overall health of animals (Teng and Kim). Similar to prebiotics, different probiotics alone or in combination with other additives like enzymes have also been tried to improve intestinal health and nutrient utilization $(3,22)$. Duarte et al. evaluated the symbiotic effect of prebiotic (Bacillus sp.) and xylanase enzyme in E. coli F18+ challenged weaned pigs. The study found that the feed additives were able to mitigate the negative effects of $E$. coli F18+ infection in pigs fed an antibiotic-free diet and enhanced the growth performance by reducing diarrhea, boosting immune response, and managing oxidative stress in the jejunum. In addition, Ma et al. found that synbiotic supplementation in the maternal diet positively affects the gut health of piglets, including improving nutrient metabolism, reducing oxidative stress, and improving intestinal barrier permeability function. Similarly, Grosu et al. used grapeseed meal (GSM) with bioactive compounds (such as polyphenols, PUFA, DF, minerals, etc.) in pig diets. They found that the grapeseed meal had a selective modulatory effect on several bacterial genera in the colon of pigs challenged with dextran sodium sulfate, as a model for inflammatory bowel diseases, suggesting that the GSM can be used as a potential anti-inflammatory additive in weaned piglets.

In conclusion, there are different dietary components and feed additives that can be used to modulate the intestinal health 
and functions of young monogastric animals. It can be a tool for nutritionists to develop a feeding program in the postantibiotic era. However, types, forms, and dose levels of these dietary components and additives need to be considered to obtain optimum benefits.

\section{REFERENCES}

1. Kim SW, Less JF, Wang L, Yan T, Kiron V, Kaushik SJ, et al. Meeting global feed protein demand: challenge, opportunity, and strategy. Annual Rev Anim Biosci. (2019) 7:17.1-17.23. doi: 10.1146/annurev-animal-030117-014838

2. Kim SW. Meeting amino acid requirements in pig nutrition. In: Wiseman J, editor. Achieving Sustainable Production of Pig Meat Vol 2. Animal Breeding and Nutrition. Sawston: Burleigh Dodds Science Publishing (2017). p. 145-65.

3. Jha R, Das R, Oak S, Mishra P. Probiotics (direct-fed microbials) in poultry nutrition and their effects on nutrient utilization, growth and laying performance, and gut health: a systematic review. Animals. (2020) 10:1863. doi: 10.3390/ani10101863

4. Jha R, Berrocoso JD. Review: dietary fiber utilization and its effects on physiological functions and gut health of swine. Animal. (2015) 9:144152. doi: 10.1017/S1751731115000919

5. Yadav S, Jha R. Strategies to modulate the intestinal microbiota and their effects on nutrient utilization, performance, and health of poultry. J Anim Sci Biotechnol. (2019) 10:2. doi: 10.1186/s40104-018-0310-9

6. Jang K, Kim SW. Supplemental effects of dietary nucleotides on intestinal health and growth performance of newly weaned pigs. J Anim Sci. (2020) 97:4875-82. doi: 10.1093/jas/skz334

7. Shen YB, Perket P, Park I, Malheiros RD, Kim SW. Effects of feed grade Lmethionine on intestinal redox status, intestinal development, and growth performance of young chickens compared with conventional DL-methionine. J Anim Sci. (2015) 93:2977-86. doi: 10.2527/jas.2015-8898

8. Tiwari UP, Chen H, Kim SW, Jha R. Supplemental effect of xylanase and mannanase on nutrient digestibility and gut health of nursery pigs studied using both in vivo and in vitro model. Anim Feed Sci Technol. (2018) 245:7790. doi: 10.1016/j.anifeedsci.2018.07.002

9. Duarte ME, Zhou F, Dutra W, Kim SW. Dietary supplementation of xylanase and protease on performance, digesta viscosity, nutrient digestibility, immune and oxidative stress status and gut health of newly weaned pigs. Anim Nutr. (2020) 5:351-8. doi: 10.1016/j.aninu.2019.04.005

10. Choct M. Managing gut health through nutrition. Br Poult Sci. (2009) 50:915. doi: 10.1080/00071660802538632

11. Kim SW, Holanda DM, Gao X, Park IK, Yiannikouris A. Efficacy of a yeast cell wall extract to mitigate the effect of naturally cooccurring mycotoxins contaminating feed ingredients fed to young pigs: impact on gut health, microbiome, and growth. Toxins. (2020) 11:E633. doi: 10.3390/toxins11110633

12. Passos AA, Park I, Ferket P, von Heimendahl E, Kim SW. Effect of dietary supplementation of xylanase on apparent ileal digestibility of nutrients, viscosity of digesta, and intestinal morphology of growing pigs fed corn and soybean meal based diet. Anim Nutr. (2015) 1:3640. doi: 10.1016/j.aninu.2015.02.006

\section{AUTHOR CONTRIBUTIONS}

All authors listed have made a substantial, direct and intellectual contribution to the work, and approved it for publication.

13. Chen H, Zhang S, Park I, Kim SW. Effects of supplemental protease on growth performance, nutrient digestibility, and gut health in nursery pigs fed diets with corn or sorghum. Anim Nutr. (2017) 3:35965. doi: 10.1016/j.aninu.2017.09.005

14. Sun Y, Kim SW. Intestinal challenge with enterotoxigenic Escherichia coli in pigs, and nutritional intervention to prevent postweaning diarrhea. Anim Nutr. (2017) 3:322-30. doi: 10.1016/j.aninu.2017.10.001

15. Adhikari P, Kiess A, Adhikari R, Jha R. An approach to alternative strategies to control avian coccidiosis and necrotic enteritis. J Appl Poult Res. (2020) 29: 515-34. doi: 10.1016/j.japr.2019.11.005

16. Yegani M, Korver DR. Factors affecting intestinal health in poultry. Poult Sci. (2008) 87:2052-63. doi: 10.3382/ps.2008-00091

17. Berrocoso JD, Kida R, Singh AK, Kim YS, Jha R. Effect of in ovo injection of raffinose on growth performance and gut health parameters of broiler chicken. Poult Sci. (2017) 96:1573-80. doi: 10.3382/ps/pew430

18. Zhang J, Cai $\mathrm{K}$, Mishra $\mathrm{R}$, Jha $\mathrm{R}$. In ovo supplementation of chitooligosaccharide and chlorella polysaccharide affect cecal microbial community, metabolic pathways, and fermentation metabolites in broiler chickens. Poult Sci. (2020) 99:4476-785. doi: 10.1016/j.psj.2020.06.061

19. Adhikari B, Kim SW, Kwon YM. Characterization of microbiota associated with digesta and mucosa in different regions of gastrointestinal tract of nursery pigs. Int J Mol Sci. (2019) 20:1630. doi: 10.3390/ijms20071630

20. Passos AA, Andrade C, Phillips CE, Coffey MT, Kim SW. Nutrient value of spray field forages fed to pigs and the use of fibrolytic enzymes to enhance nutrient digestibility. J Anim Sci. (2015) 93:1721-8. doi: 10.2527/jas201 4-8435

21. Niu Q, Li P, Hao S, Zhang Y, Kim SW, Li H, et al. Dynamic distribution of the gut microbiota and the relationship with apparent crude fiber digestibility and growth stages in pigs. Sci Rep. (2015) 5:9938. doi: 10.1038/srep09938

22. Singh AK, Tiwari UP, Berrocoso JD, Dersjant-Li Y, Awati A, Jha R. Effect of a combination of xylanase, amylase, and protease and probiotics on major nutrients including amino acids and non-starch polysaccharides utilization in broilers fed different level of fibers. Poult Sci. (2019) 98:557181. doi: $10.3382 / \mathrm{ps} /$ pez310

Conflict of Interest: The authors declare that the research was conducted in the absence of any commercial or financial relationships that could be construed as a potential conflict of interest.

Copyright (c) 2021 Jha and Kim. This is an open-access article distributed under the terms of the Creative Commons Attribution License (CC BY). The use, distribution or reproduction in other forums is permitted, provided the original author(s) and the copyright owner(s) are credited and that the original publication in this journal is cited, in accordance with accepted academic practice. No use, distribution or reproduction is permitted which does not comply with these terms. 